\title{
Class-E Oscillators as Wireless Power Transmitters for Biomedical Implants
}

\author{
Anthony N. Laskovski \\ School of Electrical Engineering and \\ Computer Science \\ The University of Newcastle \\ Callaghan, NSW, Australia 2308 \\ Email: alasko@ieee.org
}

\author{
Mehmet R. Yuce \\ School of Electrical Engineering and \\ Computer Science \\ The University of Newcastle \\ Callaghan, NSW, Australia 2308 \\ Email: Mehmet.Yuce@newcastle.edu.au
}

\begin{abstract}
This paper presents the use of Class-E oscillators as inductive power transmitters for implanted telemetry devices that transmit information sensed by biosensors. Several Class-E oscillators are compared with an equivalent Class-E amplifier, showing higher efficiency while maintaining frequency clarity, stability and accuracy. Energy has been successfully transferred to a receiving inductor $1.5 \mathrm{~cm}$ away, which has been rectified to produce a $1 \mathrm{~V}_{D C}$ signal.
\end{abstract}

\section{INTRODUCTION}

Wireless power transmitters form an important role in supplying energy to implanted electronic devices and biosensors. Inductive coupling was introduced to biomedical implants to recharge implanted batteries for devices such as pacemakers in order to avoid periodic surgery to replace flat batteries [1] [2].

As biomedical implant technology progresses with developments such as cochlear and retinal prosthesis, attention is increasingly being focused on the supply of constant wireless power within tight space and power constraints. As space restrictions tighten, so does the allowable size of components in the implanted environment. This implies that higher frequencies of operation are required such that the size of circuit components is reduced. However, the transfer of energy to implanted devices becomes less efficient as the frequency of transmission increases [3]. This makes the design of highly efficient higher frequency power transmitters a point of interest.

The concepts behind inductive power transfer may be understood by considering a weakly coupled transformer, with a primary coil from which energy is transmitted and a secondary coil at which energy is received. The core of this transformer is a combination air and several layers of human tissue between the two coils. Once the energy is received at the secondary coil, it is rectified and regulated as a DC supply for the implanted electronics and biosensors. The primary coil external to the body is driven by a power transmitter.

Given that space restrictions cause a natural progression towards smaller coils and therefore higher transmission frequencies, power transmitters have been developed at higher frequencies. One particular field of interest has been switching power amplifiers. They operate efficiently at higher frequen-

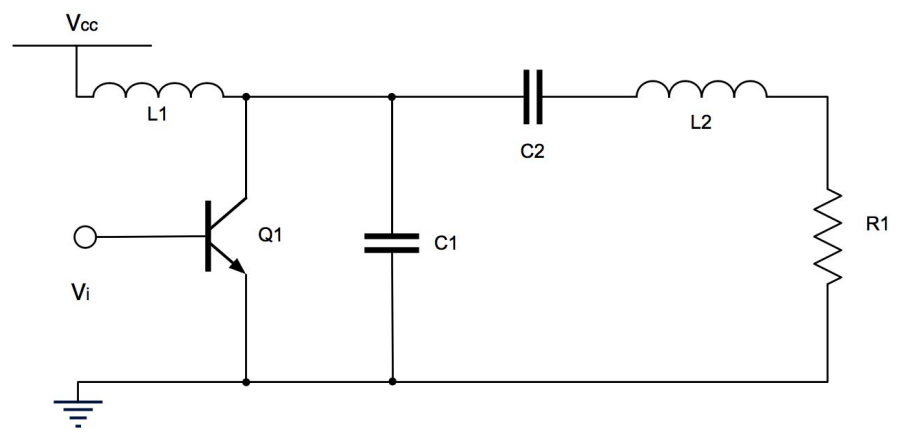

Fig. 1. The Class-E amplifier [5]

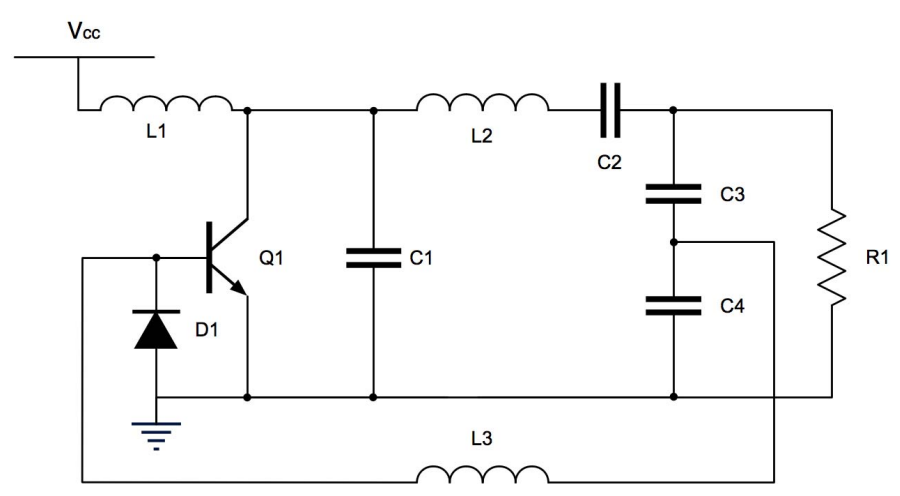

Fig. 2. The Class-E oscillator [6]

cies, which is why the Class-E power amplifier has been widely adopted as the means by which energy is inductively transferred to an implanted device [4].

Shown in Fig. 1, the Class-E amplifier comprises an inductor $L_{2}$. This inductor represents the primary coil for the transmission of wireless energy to an implanted device. The amplifier's efficiency at high frequencies is attributed to its ability to hold zero charge across the terminals of the transistor while it is switching. Not only is the voltage designed to be zero, but so is the rate of change of voltage $(d v / d t)$. It allows for a power amplifier which efficiently transmits energy across $L_{2}$ according to the switching frequency supplied by the input voltage $v_{i}$. The capacitor $C_{1}$ also absorbs parasitic 
capacitance that exists between transistor terminals, which becomes increasingly significant at higher frequencies where design parameters are in the order of parasitic impedances [5], [7], [8].

The Class-E amplifier works efficiently at high frequencies, however the fact that it is a switching power amplifier means that it requires a high frequency square-wave input in order to operate effectively. This assumption does not consider the energy that is required to produce the square-wave input using a dedicated oscillator, be it a crystal or LC oscillator.

The idea of feeding an oscillated output signal back to the input of the amplifier implies that the circuit would be selfoscillating, similar to the commonly known Colpitts oscillator, while operating with zero switching conditions. This is the concept behind the Class-E oscillator as shown in Fig, 2 [6].

Additional circuit elements have been added to form the Class-E oscillator, namely the feedback elements $C_{3}, C_{4}$ and $L_{3}$. It was designed by Ebert et al. to constructively shift the phase of the feedback point of the oscillator [6]. The diode $D_{1}$ is placed at the input of the transistor in order to clip the input signal such that it appears as a square wave, satisfying the requirement of the Class-E circuit to have a square-wave input.

Given that low power consumption is advantageous in biomedical systems, it is useful to consider a self oscillating Class-E oscillator as a wireless power transmitter rather than a Class-E power amplifier. Similar to the power amplifier, the oscillator would transmit energy through $L_{2}$.

\section{Design and Implementation of Class-E TRANSMITTERS}

\section{A. Comparison of Class-E Topologies}

In order to determine whether using a Class-E oscillator is advantageous over a Class-E amplifier, similarly designed circuits have been constructed and tested with the main circuit elements chosen to be as similar as possible. The transistor used for all of the five circuits is BC547B from Fairchild Semiconductors. The elements of the Class-E circuits have been determined according to [8], and all of the circuits are supplied with a $3 \mathrm{~V}$ supply. The inductor $L_{2}$ is in fact a spiral inductor, forming the primary coil for the transfer of inductively transferred power.

The first power transmitter circuit uses the Class-E amplifier, as shown in Fig.3. The 27-MHz clock input signal to the amplifier is produced by the crystal oscillator ACHL-27MHZEK. Small $1 \Omega$ measurement-resistors $R_{\text {meas }}$ have been positioned to measure the total current and current through the transmission coil such that the input and output power can be measured. The $27 \mathrm{MHz}$ frequency of transmission is in the allowable limits of the Industrial, Scientific and Medical (ISM) band.

The power used by the circuit is $77 \mathrm{~mW}(18.9 \mathrm{dBm})$, which is determined by multiplying the supply voltage with the r.m.s. value of the total current. The output power of $11.2 \mathrm{dBm}$ is calculated by multiplying the r.m.s. voltage and current across

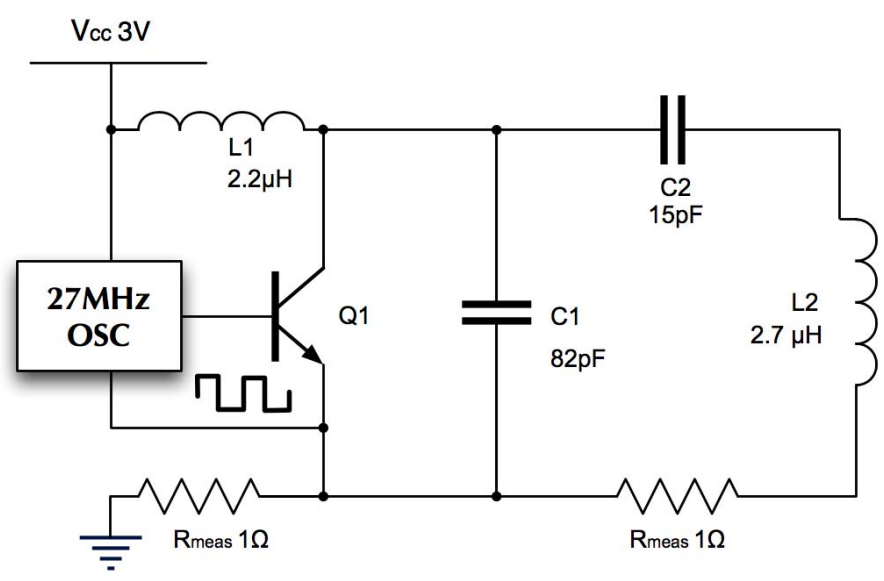

Fig. 3. A $27 \mathrm{MHz}$ Class-E amplifier constructed in hardware, driven by a crystal oscillator.

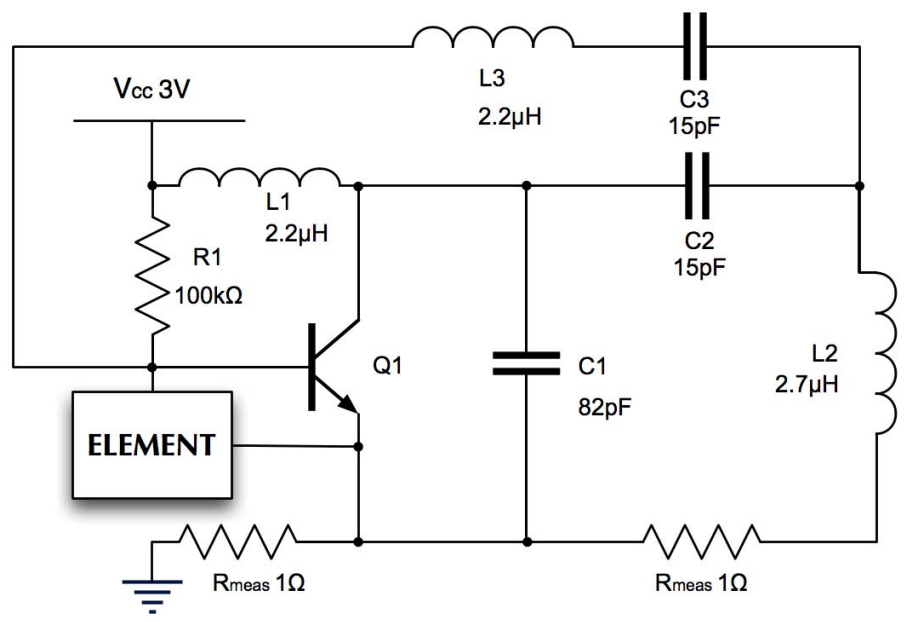

Fig. 4. Schematic diagram of a Class-E oscillator with an LC feedback network and element between the transistors base and emitter terminals, being either a $100 \mathrm{k} \Omega$ resistor or a Schottky diode.

and through the inductor $L_{2}$. This corresponds to an efficiency of $17 \%$.

The next step of the comparison involves the construction of the Class-E oscillator circuit, shown in Fig.4. This differs from the Class-E oscillator shown in Fig.2 in that the load is represented by the inductor $L_{2}$ rather than a resistor. The feedback point is also taken between $L_{2}$ and $C_{2}$, and consists of an LC pair in order to reverse the phase difference that is incurred at the point across $L_{2}$. The element between the base and emitter of $Q_{1}$ is a $100 \mathrm{k} \Omega$ biasing resistor. The power used by this circuit is $35 \mathrm{~mW}(15.5 \mathrm{dBm})$ and $11.4 \mathrm{dBm}$ is transmitted, corresponding to an efficiency of $39 \%$. While this is much higher than the efficiency of the Class-E Amplifier of Fig. 3 , the $34 \mathrm{MHz}$ frequency of the output is higher than the desired $27 \mathrm{MHz}$ value. This is due to the fact that the accuracy of the circuit's frequency is controlled solely by the accuracy of its individual inductor and capacitor values.

A variant of this circuit has been implemented by inserting a 27MHz crystal (Citizen America CS1027.000MABJ-UT) as 


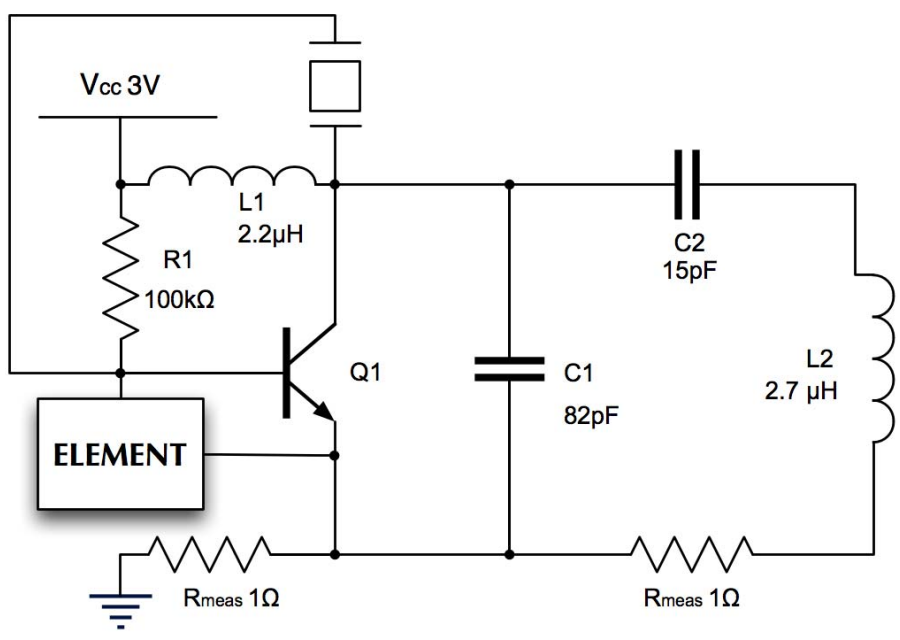

Fig. 5. Schematic diagram of a Class-E oscillator with a $27 \mathrm{MHz}$ Crystal feedback network and element between the transistors base and emitter terminals, being either a $100 \mathrm{k} \Omega$ resistor or a Zener diode.

the feedback network in order to create a stable frequency. This crystal is not an oscillator itself, but an accurate impedance that ensures the input frequency to the transistor is $27 \mathrm{MHz}$. The schematic of this circuit is shown in Fig.5, with the baseemitter element being $100 k \Omega$ resistor. The power used by the circuit has been measured to be $112 \mathrm{~mW}(20.5 \mathrm{dBm})$ and the output power is also $20.5 \mathrm{dBm}$, which corresponds to a measured efficiency of $100 \%$. This is attributed to negligibly small losses in the biasing circuit of the amplifier and zeroswitching conditions at the collector of the transistor.

\section{B. Class-E Oscillators biased with Diodes}

Class-E oscillators presented in literature have employed Schottky diodes across the base and emitter of the circuit's transistors [6]. This is usually done to clip the input signal such that it approximates a square-wave input, which is preferred as the transistor continues to operate as a switch.

The LC feedback Class-E oscillator of Fig.4 has been implemented using a Schottky diode from Fairchild Semiconductors (MBR0520L), which is the same diode used in [6]. The power used by this circuit is $42 \mathrm{~mW}(16.2 \mathrm{dBm})$ and an output power of $9.9 \mathrm{dBm}$ is produced, corresponding to an efficiency of $23 \%$, which is considerably lower than the $39 \%$ efficiency achieved with a $100 k \Omega$ resistor in place of the diode. Zener diodes are a more suitable option than the Schottky diode used, however the voltage level of the output is not high enough to switch the transistor.

A 3V Zener diode (NXP-BZX384-C3V0) has been implemented as the element between the base and emitter of $Q_{1}$ in a Class-E oscillator based on Fig.5. The circuit works in that the feedback voltage is high enough to drive the transistor and Zener diode. The circuit's power usage has been recorded as $63 \mathrm{~mW}(18 \mathrm{dBm})$, with an output power of $18 \mathrm{dBm}$. This mathematically corresponds to $100 \%$ efficiency, and is attributed to negligibly small losses in the biasing circuit of

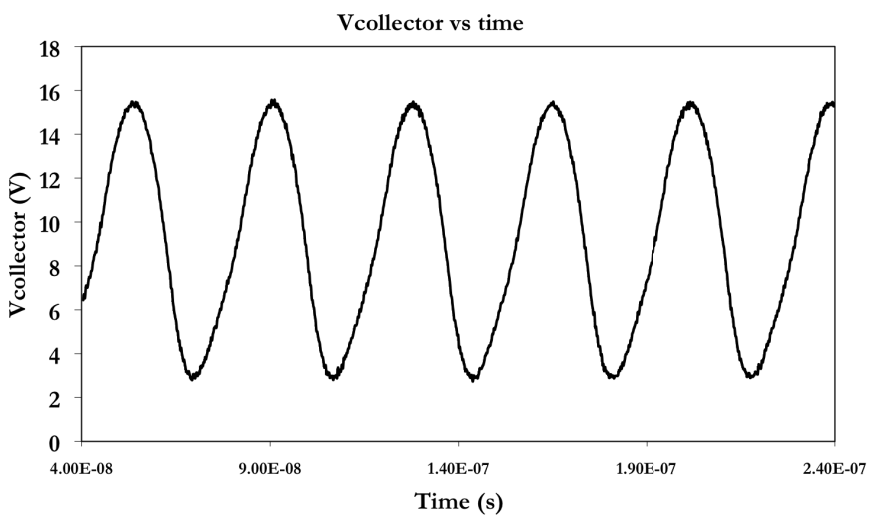

Fig. 6. Voltage at the collector of Q1 in Fig. 5 with a 9V supply and a $3 \mathrm{~V}$ Zener diode between the base and emitter.

the amplifier and zero-switching conditions at the collector of the transistor.

The results of these experiments are summarised in Table I. The first significant point is that using a feedback network in the Class-E circuit improves the efficiency of the transmitter, in that a dedicating oscillating unit is no longer required.

Another significant point is that including a crystal in the feedback network allows the frequency of the transmitter to be controlled, rather than relying on the accuracy of individual inductors and capacitors, which may cause unstable or inaccurate transmission frequencies.

The use of zener diodes on the base of the circuit's transmitter makes little difference on the performance of the amplifier, however it may be an important to regulate the output of the transmitter in the case that the feedback voltage becomes too high.

\section{Class-E Oscillator with Crystal Feedback}

A higher-power Class-E oscillator based on Fig.5 has been tested, operating at $9 \mathrm{~V}$ and using the Zener diode (NXPBZX384-C3V0). The voltage at the collector of the transistor is shown in Fig.6, which ranges from $3 \mathrm{~V}$ to over $15 \mathrm{~V}$. Ideally the lowest point should be $0 \mathrm{~V}$, as this would remove the wasted energy discharged on the capacitor $C_{1}$.

The input voltage at the transistor is shown in Fig.7, where the intended function of the Zener diode can be seen as the input signal is clipped at $3 \mathrm{~V}$. The output voltage across $V_{L_{2}}$ is shown in Fig.8, measuring at $18 \mathrm{~V}$. As indicated by the waveform, it is a reasonably clean $27 \mathrm{MHz}$ sinusoidal wave.

The input power used by this circuit measures at $794 \mathrm{~mW}$ $(29 \mathrm{dBm})$, with an output power of $28 \mathrm{dBm}$. This corresponds to a measured efficiency of $80 \%$. The likely cause of the circuit's deviation from $100 \%$ efficiency is the voltage at the collector not reaching zero during the transistor's switching points, causing a discharge of energy from $C_{1}$.

\section{Wireless Power Transfer}

The higher-power Class-E oscillator of Section II-C has been tested as a wireless power transmitter, with $L_{2}$ rep- 
TABLE I

Comparisons BetWeEn Class-E CiRCUITS

\begin{tabular}{llrrrr}
\hline Class-E Circuit & B/E Element & $f$ & Power Use & V $_{L_{2} p p}$ & Output Power \\
\hline Amplifier of Fig.3 & & $27 \mathrm{MHz}$ & $18.9 \mathrm{dBm}$ & $1.6 \mathrm{~V}$ & $11.2 \mathrm{dBm}$ \\
Oscillator with LC feedback, Fig.4 & $100 \mathrm{k} \Omega$ & $34 \mathrm{MHz}$ & $15.5 \mathrm{dBm}$ & $0.9 \mathrm{~V}$ & $11.4 \mathrm{dBm}$ \\
Oscillator with Crystal Feedback, Fig.5 & $100 k \Omega$ & $27 \mathrm{MHz}$ & $20.5 \mathrm{dBm}$ & $7.0 \mathrm{~V}$ & $20.5 \mathrm{dBm}$ \\
Oscillator with LC feedback, Fig.4 & Schotky Diode & $34 \mathrm{MHz}$ & $16.2 \mathrm{dBm}$ & $1.2 \mathrm{~V}$ & $9.9 \mathrm{dBm}$ \\
Oscillator with Crystal Feedback, Fig.5 & Zener Diode & $27 \mathrm{MHz}$ & $18.0 \mathrm{dBm}$ & $6.7 \mathrm{~V}$ & $18.0 \mathrm{dBm}$ \\
\hline
\end{tabular}

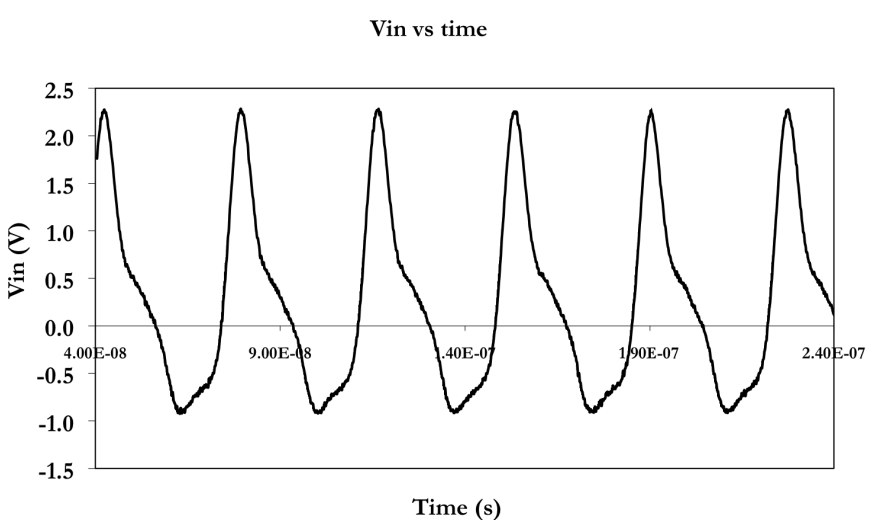

Fig. 7. Voltage at the input of Q1 in Fig. 5 with a 9V supply and a $3 \mathrm{~V}$ Zener diode between the base and emitter.

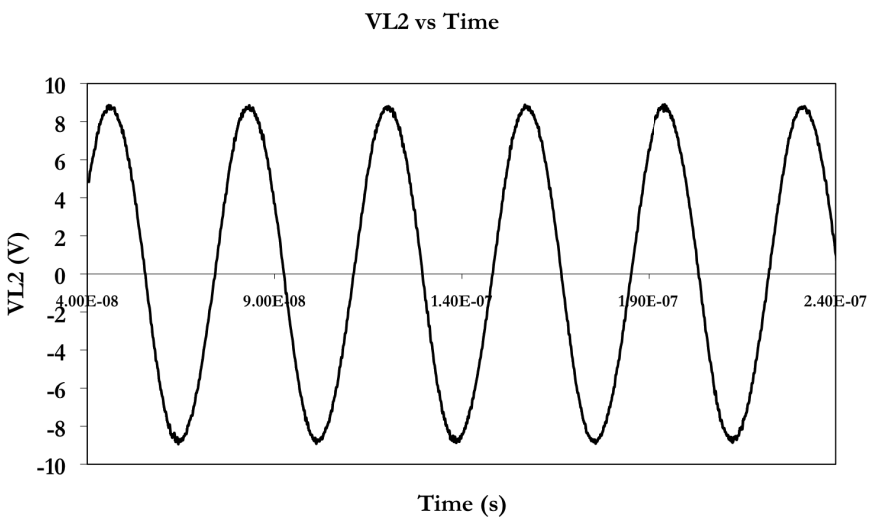

Fig. 8. Voltage across output spiral $L_{2}$ in Fig. 5 with a $9 \mathrm{~V}$ supply and a $3 \mathrm{~V}$ Zener diode between the base and emitter.

resenting the transmitter spiral. The receiving coil is the stacked spiral inductor of Fig.9, which was designed for use in biological implants.

At a distance of $1.5 \mathrm{~cm}$ a voltage of $1.5 V_{p p}$ is received on the coil. This signal has been rectified to produce the $1 \mathrm{~V}$ signal shown in Fig.11, which is a level at which current IC technologies and biosensors operate at.

\section{CONCLUSION}

This paper proposes the use of Class-E oscillators as inductive power transmitters for implanted telemetry devices that transmit information read by biosensors. Several ClassE oscillators were compared with the commonly used Class-E amplifier. The Class-E oscillators were determined to be more

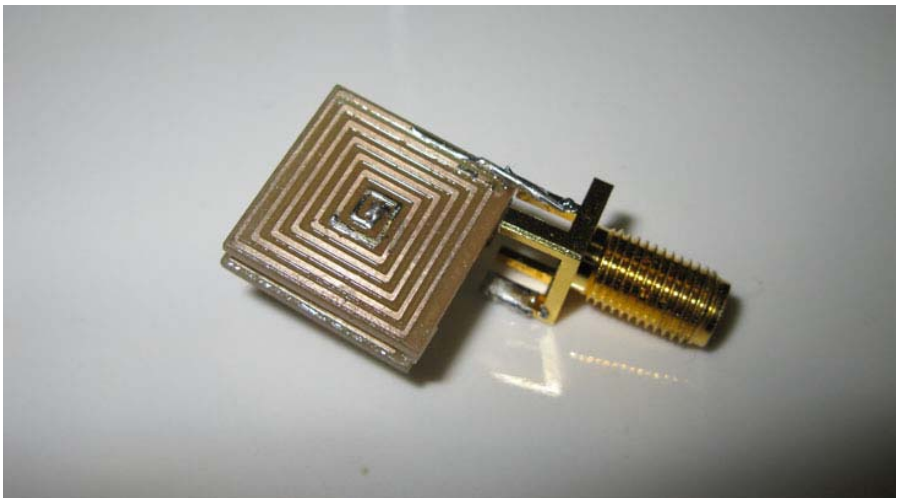

Fig. 9. Stacked spiral receiving inductor [9].

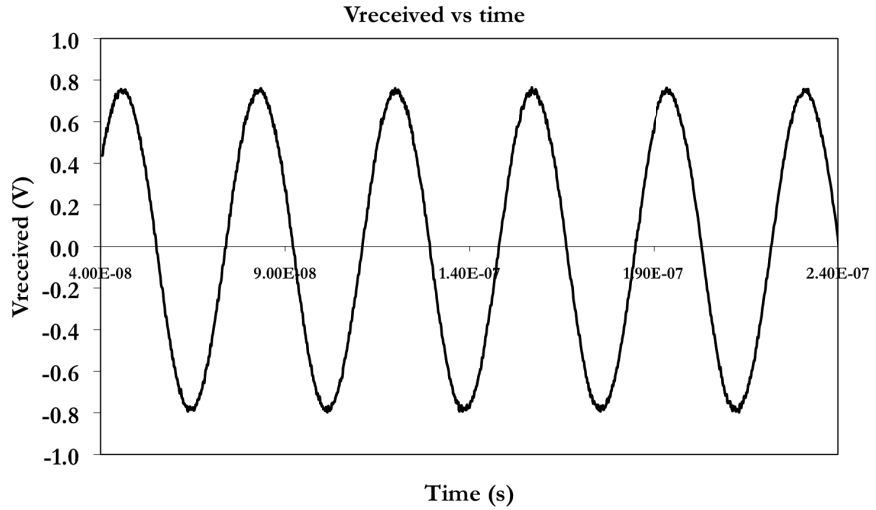

Fig. 10. Voltage received on a spiral coil from the transmitter shown in Fig. 5 with a $9 \mathrm{~V}$ supply and a $3 \mathrm{~V}$ Zener diode between the base and emitter.

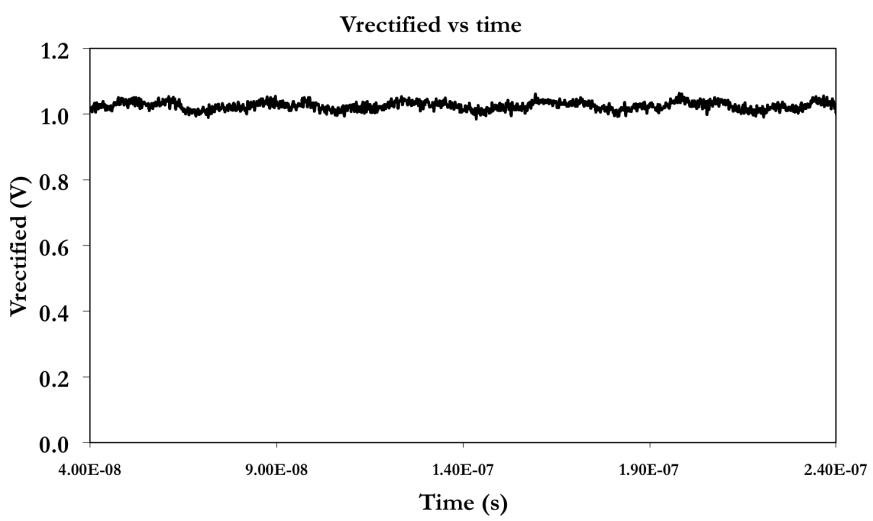

Fig. 11. Rectified voltage received on a spiral coil from the transmitter shown in Fig. 5 with a 9V supply and a 3V Zener diode between the base and emitter. 
efficient when considering the power used by the input signal to the amplifier.

Different feedback options are compared amongst Class-E oscillators, and it is proposed that a crystal feedback network ensures a more efficient circuit with a more stable and better controlled frequency. The use of Zener diodes at the input of the oscillator is determined to be advantageous when operating at higher input voltage levels, in that the input is clipped to the diode's voltage rating, allowing the Class-E load to operate as designed.

A higher-power Class-E oscillator has been constructed based on the results of the circuit comparisons, transmitting $794 \mathrm{~mW}(28 \mathrm{dBm})$ of power at $27 \mathrm{MHz}$ and operating at a frequency of $80 \%$. This was successfully received and rectified to produce a $1 \mathrm{~V}$ signal on a secondary inductor $1.5 \mathrm{~cm}$ away, which is in the useful range for biosensor applications.

\section{REFERENCES}

[1] H. Lim, Y. Yoon, C. Lee, I. Park, B. Song, and J. Cho, "Implementation of a transcutaneous charger for fully implantable middle ear hearing device," Engineering in Medicine and Biology Society, 2005. IEEE-EMBS 2005. 27th Annual International Conference of the, pp. 6813-6816, Jan 2005.

[2] P. Li and R. Bashirullah, "A wireless power interface for rechargeable battery operated medical implants," Circuits and Systems II: Express Briefs, IEEE Transactions on, vol. 54, no. 10, pp. 912-916, Oct 2007.

[3] P. Vaillancourt, A. Djemouai, J. Harvey, and M. Sawan, "Em radiation behavior upon biological tissues in a radio-frequency power transfer link for a cortical visual implant," Engineering in Medicine and Biology Society, 1997. Proceedings of the 19th Annual International Conference of the IEEE, vol. 6, pp. 2499-2502 vol.6, Oct 1997.

[4] P. Troyk and M. Schwan, "Closed-loop class e transcutaneous power and data link for microimplants," IEEE Transactions on Biomedical Engineering, vol. 39, no. 6, pp. 589-599, Jun 1992.

[5] N. Sokal and A. Sokal, "Class e-a new class of high-efficiency tuned single-ended switching power amplifiers," Solid-State Circuits, IEEE Journal of, vol. 10, no. 3, pp. 168-176, Jun 1975.

[6] J. Ebert and M. Kazimierczuk, "Class e high-efficiency tuned power oscillator," Solid-State Circuits, IEEE Journal of, vol. 16, no. 2, pp. 62 66, 1981.

[7] M. Kazimierczuk, "Class e tuned power amplifier with nonsinusoidal output voltage," Solid-State Circuits, IEEE Journal of, vol. 21, no. 4, pp. 575-581, Aug 1986.

[8] N. Sokal, "Class-e switching-mode high-efficiency tuned rf/microwave power amplifier: improved design equations," Microwave Symposium Digest., 2000 IEEE MTT-S International, vol. 2, pp. 779-782 vol.2, 2000.

[9] A. N. Laskovski, M. R. Yuce, and T. Dissanayake, "Stacked spirals for use in biomedical implants," Asia Pacific Microwave Conference, 2009. APMC 2009., pp. 389 - 392, 2009. 